\title{
Flexible Porous Carbon Black-Polymer Composites with a High Gauge Factor
}

\author{
Yi-Te Huang, ${ }^{1}$ Naoki Inomata, ${ }^{1}$ Zhuqing Wang, ${ }^{1}$ Yu-Ching Lin, ${ }^{2}$ and Takahito Ono ${ }^{3 *}$ \\ ${ }^{1}$ Department of Mechanical Systems Engineering, Tohoku University, Sendai, Miyagi 980-8579, Japan \\ ${ }^{2}$ Goertek Technology Japan Co., Ltd., Tokyo 101-0021, Japan \\ ${ }^{3}$ Micro System Integration Center, Tohoku University, Sendai 980-8579, Japan
}

(Received March 6, 2020; accepted April 14, 2020)

Keywords: nanoporous, carbon black, conductive polymer, high gauge factor, strain sensing

In this work, new conductive polymer films suitable for microfabrication have been developed. The solutions used are prepared by dispersing highly conductive nanoporous carbon blacks (n-CBs) in PHOTONEECE ${ }^{\circledR}$ (CBPh), polyvinyl alcohol (CBsPV), or gelatin (CBsGel). Conductive polymer composite thin films are formed by spin-coating on a clean glass substrate. The electrical conductivity and its response to strain are evaluated by a four-point bending method, which shows high gauge factors (GFs) of 150 and 523 for PVA- and gelatin-based composite films, respectively, in a low strain region. This clearly reveals the high potential capability of these novel nanocomposite films with the reported CB composite polymer showing a GF of $\sim 100$. It is considered that the porous structure of the $n-C B s$ provides high sensitivity of flexible sensing elements that can be implemented into microfabrication processes easily.

\section{Introduction}

Recently, research studies on microdevices for strain and pressure sensing have been extensively performed because of various potential applications,${ }^{(1-3)}$ including reliable, flexible, and sensitive strain and pressure sensors for upcoming robotic and IoT applications. ${ }^{(4-7)}$ For flexible device applications, flexible materials sensitive to strain are one of the key technologies. Among strain sensors, semiconductors, ${ }^{(8,9)}$ ceramics, ${ }^{(10)}$ and metals ${ }^{(11)}$ are often used; nevertheless, these materials are basically not flexible. Conductive polymer composites made of nonconductive polymer and conductive filler are potential candidates of flexible strain-sensitive sensing materials suitable for the microfabrication of soft strain and pressure sensors. ${ }^{(12,13)}$ Carbon black (CB) with a zero-dimensional feature is one of the carbon allotropes and is often used as a conductive filler for polymer composites. ${ }^{(14,15)}$ Carbon-based materials including carbon nanotubes and graphene have been used for improving mechanical, electrical, and thermal properties. ${ }^{(16-18)} \mathrm{CB}$ has the advantage over graphene and carbon nanotubes in terms of cost and accessibility; however, the gauge factors (GFs) of CB-based composites in strain sensing are mostly lower than $100 .{ }^{(19,20)}$ Therefore, a high-GF material based on the CB

*Corresponding author: e-mail: ono@nme.mech.tohoku.ac.jp https://doi.org/10.18494/SAM.2020.2853 
filler is desired for low-cost, accessible, and high-performance strain sensors. In this study, a highly conductive nanoporous $\mathrm{CB}$ with a smaller diameter than a conventional $\mathrm{CB}^{(21)}$ is chosen as the filler to improve GF. In this work, highly conductive nanoporous carbon black composite specimens with three types of matrix are synthesized and their GFs are evaluated.

\section{Materials and Methods}

\subsection{Conductive polymer for strain sensitivity evaluation}

The percolation effect determines the conductivity of a conductive polymer, which is the crucial point in strain sensing. A schematic structure of the CB polymer composite for the evaluation of the percolation threshold ${ }^{(22)}$ is shown in Fig. 1. The nanoporous CB particles (CB from Lion Specialty Chemicals; mean particle diameter, $34 \mathrm{~nm}$ ) in solid-state powders or in a nanoparticle solution are mixed with the polymer. As the concentration of CB increases, the resistivity of the $\mathrm{CB} /$ composite decreases abruptly after reaching the percolation threshold, at which the highest sensitivity is expected in strain sensing. ${ }^{(23,24)}$ A schematic of the test specimen is shown in Fig. 2. Porous CB particles are used as fillers, and three types of polymer are examined as the base matrixes of the composites, i.e., PHOTONEECE (Toray ${ }^{\circledR}$ ), polyvinyl

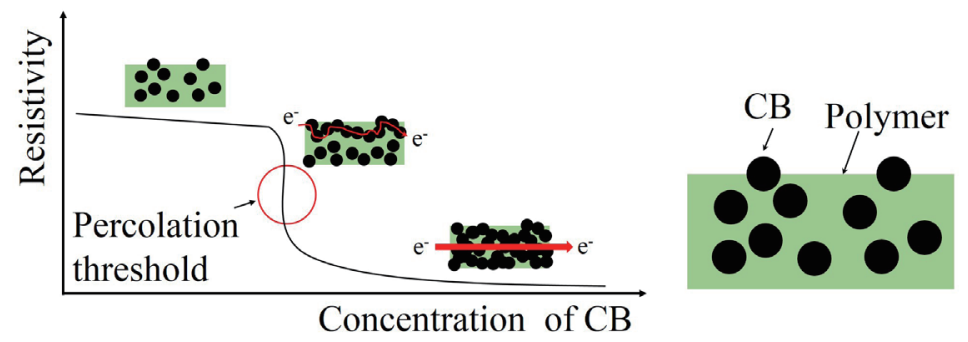

Fig. 1. (Color online) Schematic of percolation effect in conductive polymer.

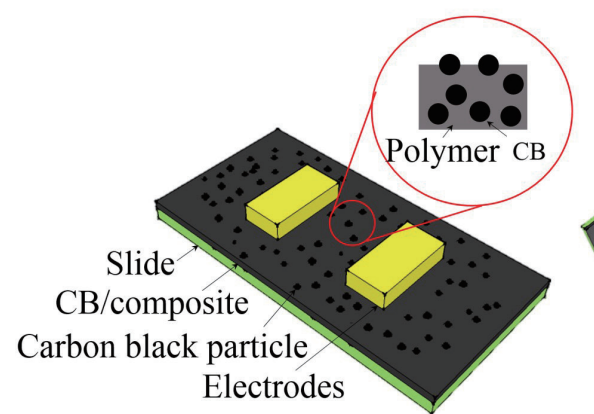

(a)

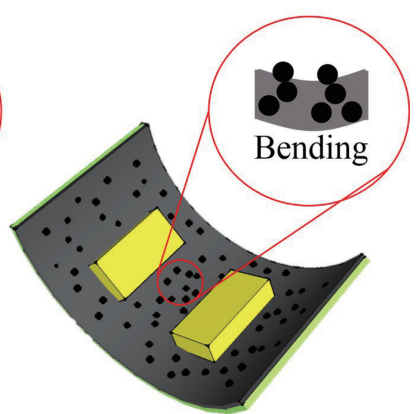

(b)

Fig. 2. (Color online) Schematic of test specimens for strain sensitivity evaluation. (a) Original and (b) bending state. 
alcohol (PVA), and gelatin. PHOTONEECE is mixed with a solid-state CB powder owing to its lack of affinity with water, while PVA and gelatin are common polymers, which are miscible in water and water-based $\mathrm{CB}$ solutions. The deformation of the $\mathrm{CB}$ polymer composites changes the distance between $\mathrm{CB}$ particles, which in turn changes the electrical conductivity. ${ }^{(25)}$ However, if the $\mathrm{CB}$ particles cannot be dispersed very well owing to aggregation, the strain sensitivity will decrease.

\subsection{Fabrication process of test specimen}

The premixing processes of the conductive polymer composites are shown in Fig. 3. N-Methyl-2-pyrrolidone (NMP) is mixed with PHOTONEECE using a planetary centrifugal mixer for 10 min, as shown in Figs. 3(a1) and 3(a2). Then, the CB powder is mixed with the PHOTONEECE solution [Fig. 3(a3)] using the planetary centrifugal mixer again for $10 \mathrm{~min}$.

For the premixing process of CBsPV, a PVA powder is poured into $95{ }^{\circ} \mathrm{C}$ hot water with stirring until the PVA powder is completely dissolved, as shown in Figs. 3(b1) and 3(b2). The CB water solution is then mixed with the PVA solution [Fig. 3(b3)] using the planetary centrifugal mixer for $10 \mathrm{~min}$.

For the premixing process of $\mathrm{CBsGel}$, a gelatin powder is added to $65{ }^{\circ} \mathrm{C}$ warm water with stirring at $800 \mathrm{rpm}$ for $20 \mathrm{~min}$, as shown in Fig. 3(c1). Glycerol is also added to gelatin [Fig. 3(c1)] to plasticize the gelatin. Then, the above solutions [Fig. 3(c2)] are mixed by rotating them for 10 min [Fig. 3(c3)]. Next, the CB solution is mixed with the gelatin solution.

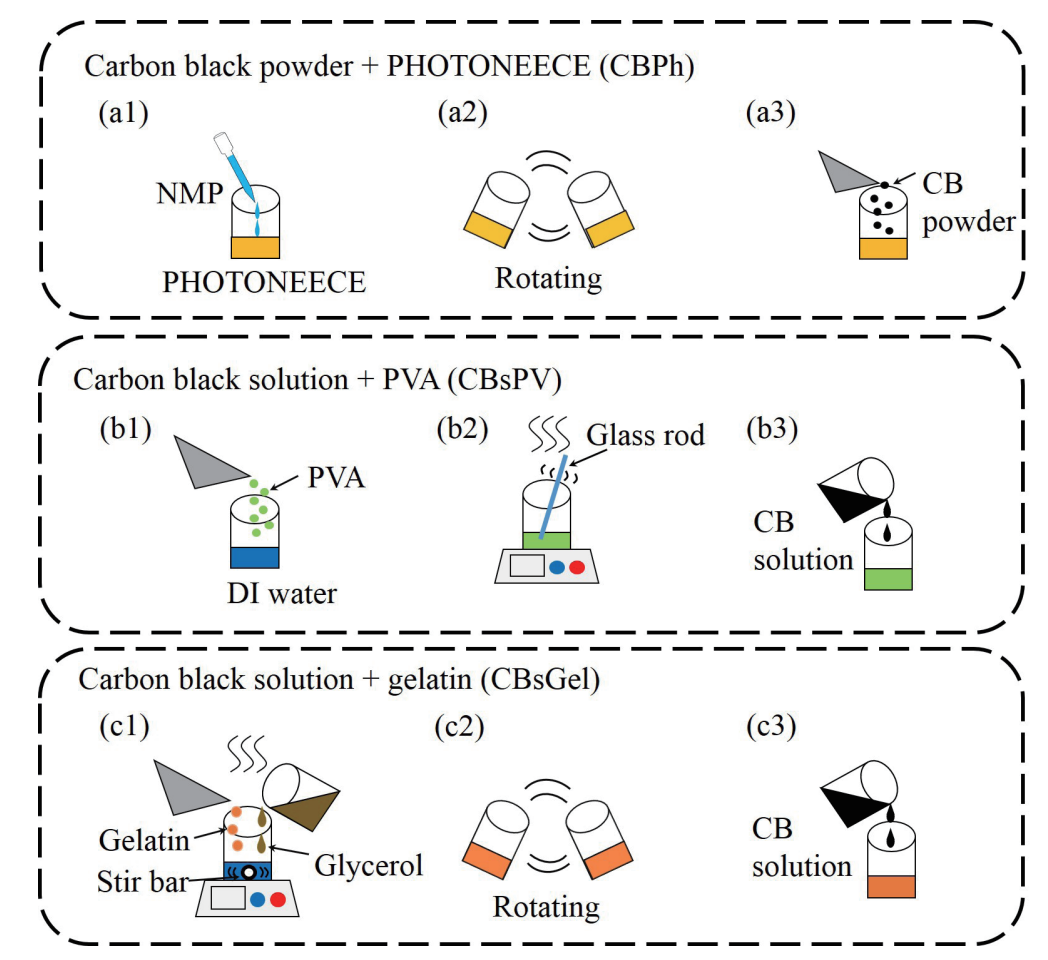

Fig. 3. (Color online) Premixing processes of the CB polymer nanocomposite films. 
Then, ultrasonication (60 min), mixing (10 $\mathrm{min})$, and vacuum pumping (60 $\mathrm{min})$ are performed to obtain a well-dispersed $\mathrm{CB}$ composite polymer. Finally, the dispersed $\mathrm{CB}$ composite polymer is spin-coated onto a $0.21 \mathrm{~mm}$ thick glass substrate at a rate of $2000 \mathrm{rpm}$ for $25 \mathrm{~s}$ to obtain a conductive polymer thin film.

\subsection{Experimental setup for evaluation of strain sensitivity to resistance}

To evaluate the intrinsic resistivity of the samples, the sheet resistance of the $\mathrm{CB}$ polymer composite thin films on the glass substrate is measured five times for each sample using a four-point probe tester (K-705RS Kyowariken ${ }^{\circledR}$ ). Through measuring the different concentrations of $\mathrm{CB}$ polymer composite thin films using the four-point probe tester, we can determine the relationship of sample sheet resistance with increasing concentration. The film thickness is measured four times for each sample from the film height using a surface profiler (P-10 Tencor ${ }^{\circledR}$ ). The average thicknesses of CBPh-, CBsPV-, and CBsGel-based composite films are approximately $1600 \pm 300,2500 \pm 600$, and $800 \pm 100 \mathrm{~nm}$, respectively. In addition, the resistivity $\rho$ can be obtained as

$$
\rho=R_{s} t
$$

where $R_{S}$ is the sheet resistance and $t$ is the thickness. The homemade experimental setup for strain sensitivity evaluation using a four-point bending measurement setup is shown in Fig. 4. The sample is electrically connected to an $I-V$ measurement system. While the sample is set on a four-point bending system with the upper load and bottom support bars, force is applied to the sample at four contact points to produce the strain calculated from the applied force. $I-V$ is measured under various force applications to evaluate the resistance variation caused by strain. In $I-V$ measurement, a voltage of $0.1 \mathrm{~V}$ is applied to the sample from $-5 \mathrm{~V}$ to $5 \mathrm{~V}$ and back and forth. The $I-V$ of each sample is measured three times at a specific strain. The resistance of the samples is evaluated from the average reciprocal of the slope of $I-V$ measurement results.

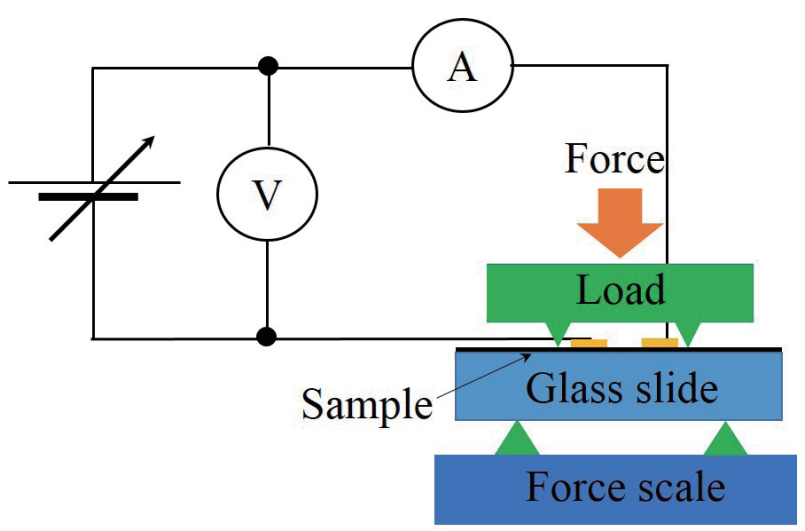

Fig. 4. (Color online) Schematic of strain sensitivity evaluation setup using four-point bending setup. 


\section{Results and Discussion}

\subsection{Formation of CB polymer composite films}

From the optical micrographs of the prepared films shown in Fig. 5, the aggregated CB particles are recognized as black spots in the PHOTONEECE matrix. This means that CB particles are not dispersed well. The aggregation of the $\mathrm{CB}$ particles in the matrix possibly causes low sensitivity to strain. As shown in Figs. 5(b) and 5(c), the CB particles are well dispersed in the PVA and gelatin matrixes. SEM images of the CB morphology of the CB polymer/composite are shown in Fig. 6. The $\mathrm{CB}$ particles on $\mathrm{CBPh}$ [Fig. 6(a)] aggregate, which indicates the inadequate dispersion of the $\mathrm{CB}$ particles. In addition, Fig. 6(b) reveals that the extent of $\mathrm{CB}$ aggregation is lower than that of $\mathrm{CBPh}$ aggregation, whereas Fig. 6(c) indicates that the extent of aggregation of the $\mathrm{CB}$ particles in gelatin is lower than that of other materials. Because of the superior miscibility of the $\mathrm{CB}$ solution in the water-based polymer solution, the $\mathrm{CB}$ particles are dispersed well in the polymer.

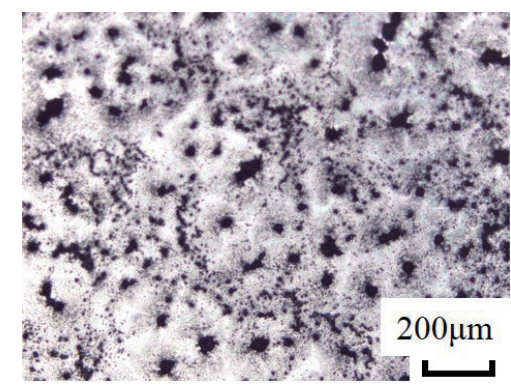

(a)

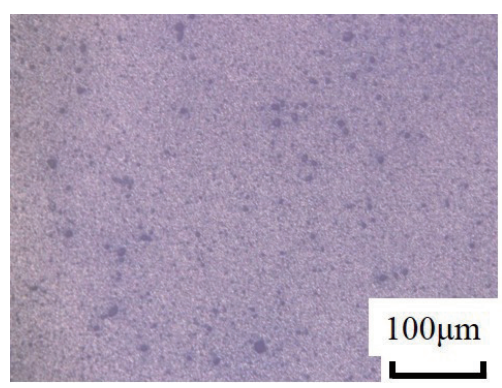

(b)

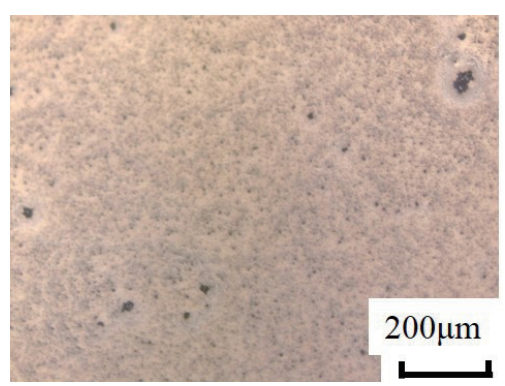

(c)

Fig. 5. (Color online) Micrographs of samples: (a) CBPh-2.28 wt\% CB, (b) CBsPV-1.60 wt\% CB, and (c) CBsGel-1.94 wt\% CB.

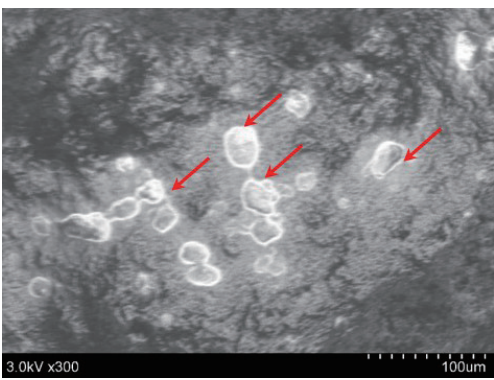

(a)

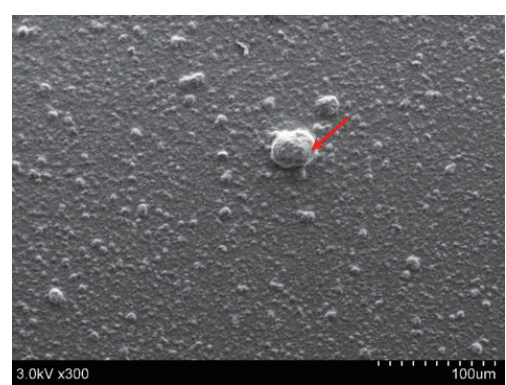

(b)

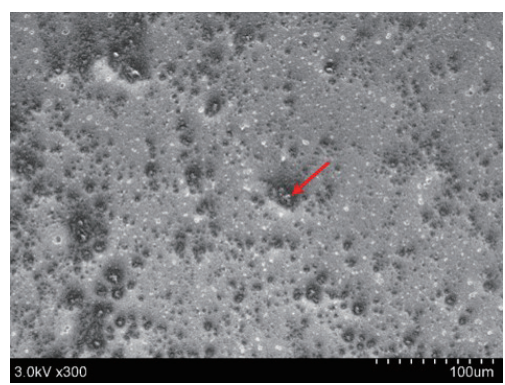

(c)

Fig. 6. (Color online) SEM images of morphology of CB on composites (a) CBPh-2.28 wt\% CB, (b) CBsPV-1.60 $\mathrm{wt} \% \mathrm{CB}$, and (c) CBsGel-1.94 wt\% CB. 


\subsection{Resistivity of CB composites}

The resistivity change of the $\mathrm{CBPh}$ composite film shows a logarithmic decrement with increasing $\mathrm{CB}$ concentration, as shown in Fig. 7. This reveals that the percolation thresholds of $\mathrm{CBPh}, \mathrm{CBsPV}$, and $\mathrm{CBsGel}$ are observed at approximately $2.3,1.6$, and $1.9 \mathrm{wt} \%$, respectively. In the next subsection, the samples with a $\mathrm{CB}$ concentration at the percolation threshold are chosen to evaluate the strain sensitivity.

\subsection{Temperature dependence of resistivity of CB polymer composites}

The temperature dependence of the samples is investigated using a temperature-controlled oven with the experimental setup shown in Fig. 8. The variation in resistance is defined as

$$
S_{\text {temp }}=\frac{\Delta R_{t}}{R_{t 0}}
$$

where $\Delta R_{t}=R_{t}-R_{t 0}$, with $R_{t}$ and $R_{t 0}$ being the resistance at a specific temperature and the resistance at room temperature $\left(20^{\circ} \mathrm{C}\right)$, respectively. The results indicate that $\mathrm{CBsGel}$ is the

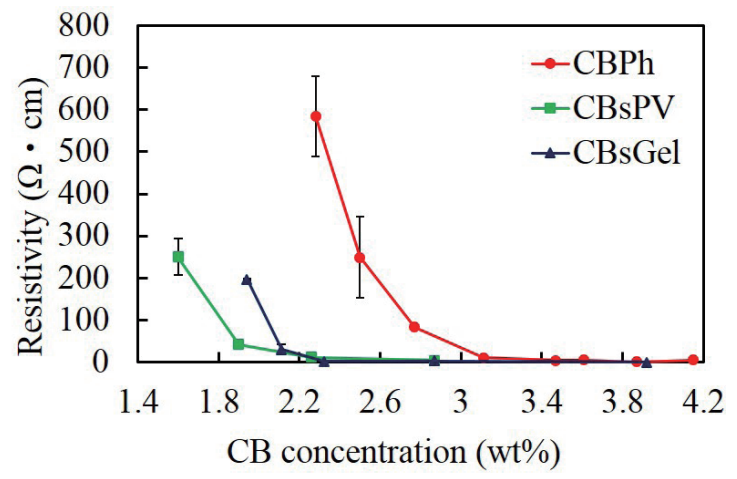

Fig. 7. (Color online) $\mathrm{CB}$ concentration dependence of resistivity of composite films.

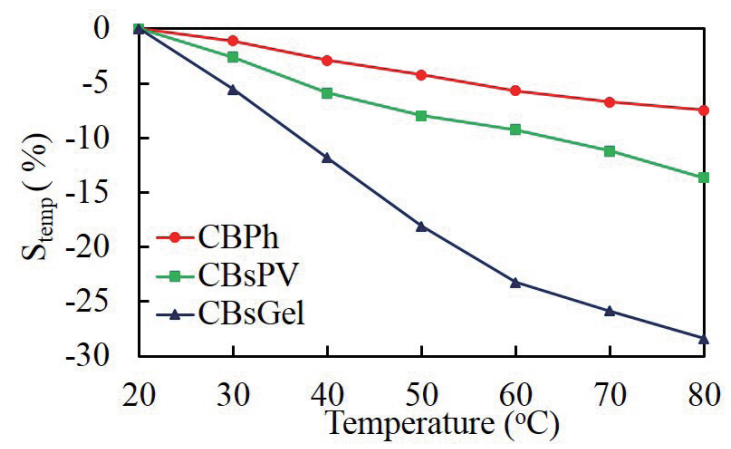

Fig. 8. (Color online) Relationship between sensitivity and temperature of (a) $\mathrm{CBPh}-2.28 \mathrm{wt} \% \mathrm{CB}$, (b) CBsPV-1.60 wt \% CB, and (c) CBsGel-1.94 wt\% CB. 
most sensitive to the variation in temperature $(-0.58 \% / \mathrm{K})$ before reaching the environmental temperature of $60{ }^{\circ} \mathrm{C}$. On the other hand, the variations in the resistances of $\mathrm{CBsPV}$ and $\mathrm{CBPh}$ are only $-0.15 \% / \mathrm{K}$ and $-0.09 \% / \mathrm{K}$ approximately before reaching the environmental temperature of $60{ }^{\circ} \mathrm{C}$, which reveals that $\mathrm{CBsPV}$ and $\mathrm{CBPh}$ are less sensitive to temperature than CBsGel. In addition, the changes in the resistances of CBsPV $(-0.15 \% / \mathrm{K})$ and $\mathrm{CBPh}$ $(-0.09 \% / \mathrm{K})$ are less than those in other work on a $\mathrm{CB}$ composite polymer strain sensor ${ }^{(25)}$ $(-0.2 \% / \mathrm{K})$. This clearly demonstrates the potential use of $\mathrm{CBsPV}$ and $\mathrm{CBPh}$ as strain sensing materials.

\subsection{Strain sensitivity of CB composites}

To estimate the strain sensitivity to the resistance of the thin films on the glass substrate, an $\mathrm{Au}$ electrode pattern is formed on the films by electron beam evaporation and photolithography, as shown in Fig. 9. Titanium $(20 \mathrm{~nm})$ is deposited on the $\mathrm{CB}$ polymer composite thin film as an adhesion layer, and a $180 \mathrm{~nm}$ gold layer is deposited on the titanium layer as the electrodes. In addition, the surface roughness of each sample is measured three times using a surface profiler (ET-200 Kosaka Laboratory ${ }^{\circledR}$ ), and the arithmetical mean roughness (Ra) is obtained as the parameter of the surface roughness of the samples. The $R a$ values of $\mathrm{CBPh}, \mathrm{CBsPV}$, and CBsGel are $0.006 \pm 0.001,0.011 \pm 0.004$, and $0.007 \pm 0.004 \mu \mathrm{m}$, respectively. This reveals that the $\mathrm{CB}$ polymer composite thin films have a roughness with a dimension of $0.001 \mu \mathrm{m}$, which is beneficial for contact with the Ti adhesion layer.

Force sensitivity is measured using the four-point bending setup, as described previously. Tensile stress is applied to the nanocomposite film and the resistance change $\Delta R$ is evaluated. The resistance of the samples subjected to different forces is obtained by $I-V$ measurement and typical results are shown in Fig. 10. Because of the good contact between the electrodes and the $\mathrm{CB}$ polymer composite thin film, the $I-V$ curves are straight in a cycle test, which also indicates a good ohmic contact between the electrodes and the CB composite polymer thin film. Similarly, a good ohmic contact is observed at high strains, which shows that the electrodes are not peeled-off from the CB polymer composite thin film by the applied strain.

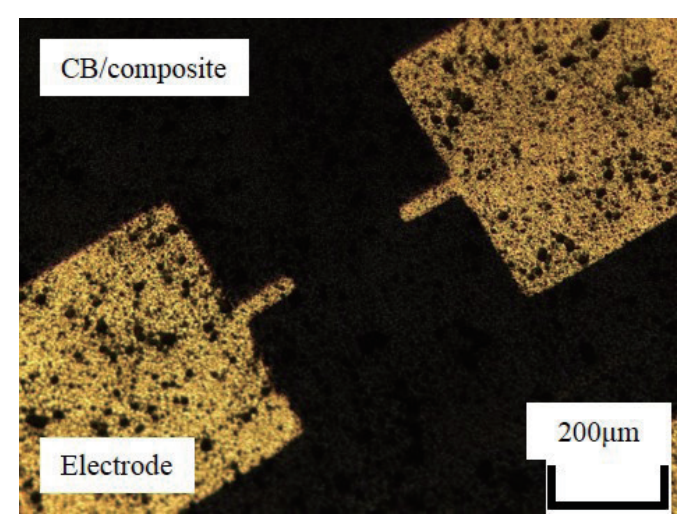

Fig. 9. (Color online) Photograph of test specimen. 


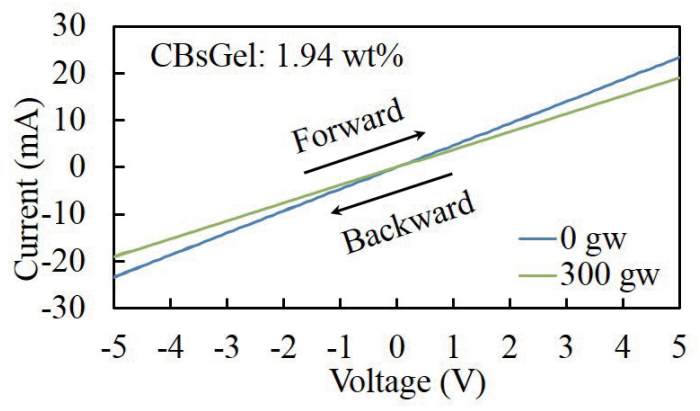

Fig. 10. (Color online) $I-V$ curve variation of CBsGel composite with various loads evaluated using four-point bending setup.

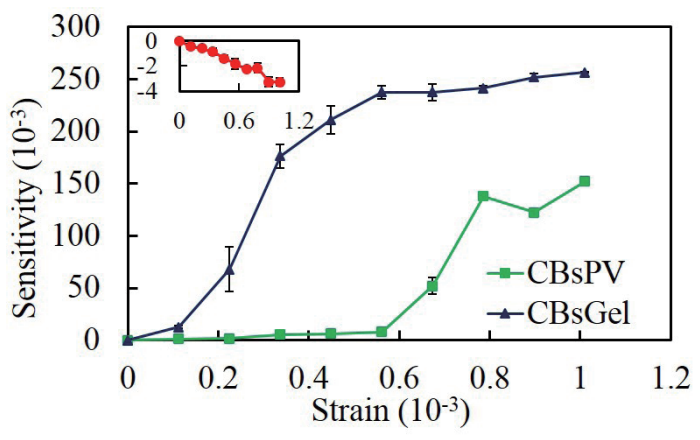

Fig. 11. (Color online) Strain dependence of sensitivity $\left(\Delta R / R_{0}\right)$ in composite films, $\mathrm{CBPh}-2.28 \mathrm{wt} \% \mathrm{CB}$ (inset), CBsPV-1.60 wt \% CB, and CBsGel-1.94 wt\% CB.

The strain sensitivity of the samples to the resistance is defined as $\Delta R / R_{0}$. The results of the strain sensitivities of the $\mathrm{CBPh}$ films with various $\mathrm{CB}$ concentrations are shown in Fig. 11. It is observed that the sensitivity does not show a linear relationship in the measured strain region. However, a high sensitivity is observed in a limited region, Here, we discuss the highest sensitivity performance. The CBPh composite film exhibits the lowest sensitivity performance, and the strain sensitivities of CBsPV and $\mathrm{CBsGel}$ composite films are much higher than that of the $\mathrm{CBPh}$ composite. The reason is that the aggregation of the $\mathrm{CB}$ particles in PHOTONEECE reduces the strain sensitivity. On the other hand, the strain sensitivity performance measured using the two-probe sensing setup may degrade owing to the contact resistance between the electrodes and the CB polymer composite thin film.

Additionally, the $\mathrm{CB}$ particles in an aqueous solution are miscible in other water-based solutions. Thus, the $\mathrm{CB}$ particles can be well dispersed in the polymer, which enhances the strain sensitivity. On the other hand, the slight aggregation of the $\mathrm{CB}$ particles in PHOTONEECE causes the low sensitivity to strain. Because of the limited dispersion, the resistance of the $\mathrm{CBPh}$ film decreases with increasing applied force. Figure 12 shows the variation in the resistance of the $\mathrm{CB}$ polymer composite film under cyclic strain application. 


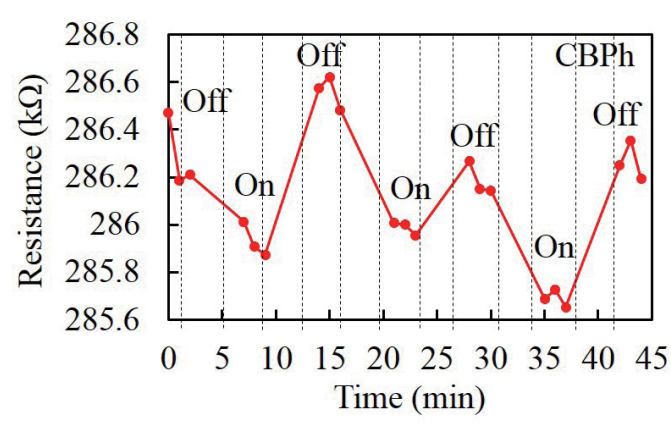

(a)

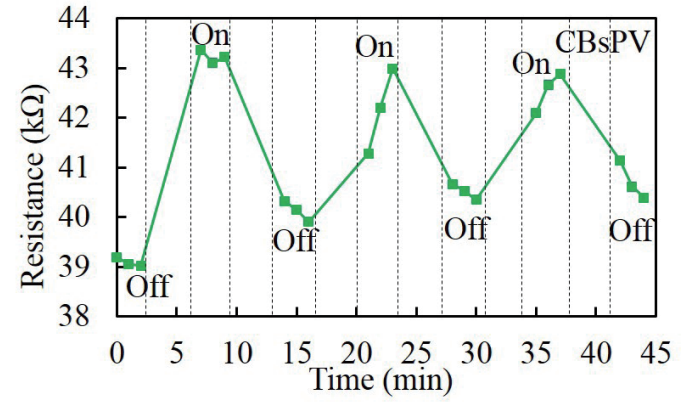

(b)

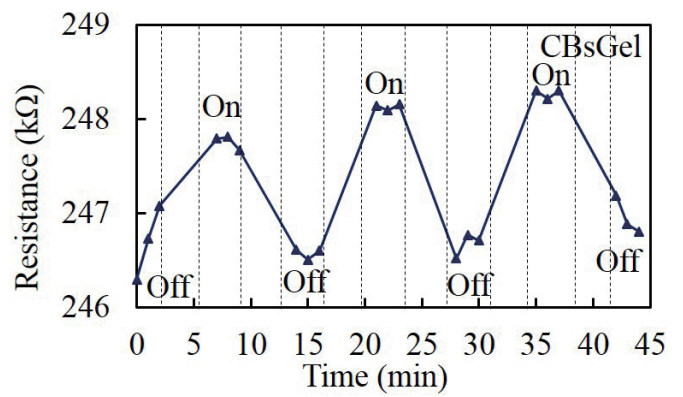

(c)

Fig. 12. (Color online) Demonstration of force sensing in composite films (a) CBPh-2.28 wt\% CB, (b) CBsPV-1.60 wt $\% \mathrm{CB}$, and (c) CBsGel-1.94 wt \% CB.

Table 1

Summarized results of the strain sensitivity.

\begin{tabular}{lccc}
\hline Sample & A & B & C \\
\hline GF & -3.3 & 150 & 523 \\
CB particles & Powder & Solution & Solution \\
Polymer & PHOTONEECE & PVA & Gelatin \\
Percolation threshold & $\sim 2.3 \mathrm{wt} \% \mathrm{CB}$ & $\sim 1.6 \mathrm{wt} \% \mathrm{CB}$ & $\sim 1.9 \mathrm{wt} \% \mathrm{CB}$ \\
\hline
\end{tabular}

Force is applied to the upper load perpendicularly (On-state) and removed (Off-state) alternately to estimate the repeatability of measurements of the CB polymer/composite. The GFs of the samples are summarized in Table 1, clearly indicating that the CBsPV and CBsGel composite films show much higher GFs of 150 and 523, respectively, which are higher than those in other works regarding $\mathrm{CB}$ composites $(\mathrm{GF}<100) .{ }^{(19,20)}$ This reveals the high potential of applying CBs to low-cost, simple-process, and flexible strain sensors based on a flexible conductive polymer. Currently, the reason for the saturation in the sensitivity at the high-strain region is not clear; but basically, the percolation effect on the resistivity is a nonlinear phenomenon, which possibly affects the nonlinearity of sensitivity. 


\section{Conclusions}

In this work, three types of $\mathrm{CB}$ polymer composite are synthesized by dispersing highly conductive porous nanostructured CB in PHOTONEECE, PVA, and gelatin. The CB particles can be dispersed well in PVA and gelatin. The CB concentration dependence on the resistivity shows a logarithmic linear relationship, also indicating the percolation effect of the $\mathrm{CB}$ particles in these polymers. Among the specimens, the $\mathrm{CBsPV}$ and $\mathrm{CBsGel}$ composite films at the percolation threshold exhibit the highest GFs of 150 and 523, respectively. These results show the potentially high capability of the $\mathrm{CBsPV}$ composite film as a strain and force sensing element with a simple process, low cost, and flexibility to detect a small strain or force.

\section{Acknowledgments}

Part of this research was performed at Tohoku University Micro System Integration Center and Micro/Nano-Machining Research and Education Center.

\section{References}

1 C. M. Boutry, A. Nguyen, Q. O. Lawal, A. Chortos, S. Rondeau-Gagné, and Z. Bao: Adv. Mater. 27 (2015) 6954. https://doi.org/10.1002/adma.201502535

2 H. Tian, Y. Shu, X. F. Wang, M. A. Mohammad, Z. Bie, Q. Y. Xie, C. Li, W. T. Mi, Y. Yang, and T. R. Ren: Sci. Rep. 5 (2015) 8603. https://doi.org/10.1038/srep08603

3 W. P. Eaton and J. H. Smith: Smart Mater. Struct. 6 (1997) 530.

4 K. Y. Shin, J. S. Lee, and J. Jang: Nano Energy 22 (2016) 95. https://doi.org/10.1016/j.nanoen.2016.02.012

5 A. G. P. Kottapalli, M. Asadina, J. M. Miao, G. Barbastathis, and M. S. Triantafyllou: Smart Mater. Struct. 21 (2012) 115030. https://doi.org/10.1088/0964-1726/21/11/115030

6 K. Nate and M. M. Tenteris: IEEE 24th Electrical Performance of Electronic Packaging and Systems Conf. (San Jose, USA, 2015). 171-174 https://doi.org/10.1109/EPEPS.2015.7347155

7 N. Watthanawisuth, T. Maturos, A. Sappat, and A. Tuantranont: IEEE Sensors (Busan, Korea, 2015) 1-4. https://doi.org/10.1109/ICSENS.2015.7370275

8 Y. Jiang, T. Ono, and M. Esashi: J. Micromech. Microeng. 19 (2009) 065030. https://doi.org/10.1088/0960$1317 / 19 / 6 / 065030$

9 Md. M. Hossain, M. Toda, T. Hokama, M. Yamazaki, K. Moorthi, and T. Ono: IEEE Sens. Lett. 3 (2019) 2500404. https://doi.org/10.1109/LSENS.2019.2893298

10 L. Zhang, Y. Wang, Y. Wei, W. Xu, D. Fang, L. Zhai, K. C. Lin, and L. An: J. Am. Ceram. Soc. 91 (2008) 1346. https://doi.org/10.1111/j.1551-2916.2008.02275.x

11 M. Zhu, J. Li, N. Inomata, M. Toda, and T. Ono: Appl. Phys. Express 12 (2019) 015003. https://doi. org/10.7567/1882-0786/aaf5c4

12 G. Latessa, F. Brunetti, A. Reale, G. Saggio, and A. D. Carlo: Sens. Actuators, B. 139 (2009) 304. https://doi. org/10.1016/j.snb.2009.03.063

13 S. C. B. Mannsfeld, B. C-K Tee, R. M. Stoltenberg, C. V. H-H. Chen, S. Barman, B. V. O. Muir, A. N. Sokolov, C. Reese, and Z. Bao: Nat. Mater. 9 (2010) 859. https://doi.org/10.1038/nmat2834

14 J. Huang: Adv. Polym. Tech. 21 (2002) 299. https://doi.org/10.1002/adv.10025

15 M. D. Walton, Y. S. Kim, C. J. Jan, E. P. McConnel, W. N. Everett, and J. C. Grunlan: Synth. Met. 157 (2007) 632. https://doi.org/10.1016/j.synthmet.2007.07.002

16 Z. An, M. Toda, and T. Ono: Composites Part B 95 (2016) 137. https://doi.org/10.1016/j.compositesb.2016.03.086

17 Q. Yu, T. Zhou, Y. Jiang, X. Yan, Z. An, X. Wang, D. Zhang, and T. Ono: Appl. Surf. Sci. 435 (2018) 617. https://doi.org/10.1016/j.apsusc.2017.11.169

18 Z. An, J. Li, A. Kikuchi, Z. Wang, Y. Jiang, and T. Ono: Microsyst. Nanoeng. 5 (2019) 20. https://doi. org/10.1038/s41378-019-0059-0

19 H. Xiao, H. Li, and J. Ou: Sens. Actuators, A 160 (2010) 87. https://doi.org/10.1016/j.sna.2010.04.027 
20 J. Ruhhammer, M Zens, F. Goldschmidtboeing, A. Seifert, and P. Woias: Adv. Mater. 16 (2015) 015003. https://doi.org/10.1088/1468-6996/16/1/015003

21 C. Mattmann, F. Clemens, and G. Tröster: J. Sensors 8 (2008) 3719. https://doi.org/10.3390/s8063719

22 H. J. Choi, M. S. Kim, D. Anh, S. Y. Yeo, and S. Lee: Sci. Rep. 9 (2019) 6338. https://doi.org/10.1038/s41598019-42495-1

23 S. Wang, X. Zhang, X. Wu, and C. Liu: Soft Matter 12 (2016) 845. https://doi.org/10.1039/C5SM01958C

24 D. J. Cohen, D. Mitra, K. Peterson, and M. M. Maharbiz: Nano Lett. 12 (2012) 1821. https://doi.org/10.1021/ nl204052z

25 C. Cochrane, V. Koncar, M. Lewandowski, and C. Dufour: J. Sens. 7 (2007) 473. https://doi.org/10.3390/ s7040473

\section{About the Authors}

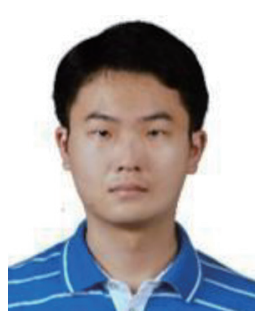

Yi-Te Huang received his B.S. degree from National Chung Hsing University, Taiwan, in 2018. Since 2018, he has pursued his M.S. degree in Tohoku University, Japan. His research interests are in MEMS, novel materials, gas sensors, strain gauge, and pressure sensing. (yite@nme.mech.tohoku.ac.jp)

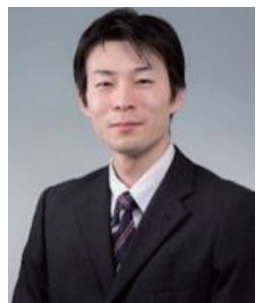

Naoki Inomata received his M.S. and D.E. degrees from Tohoku University, Sendai, Japan, in 2010 and 2013, respectively. He was an assistant professor at Micro System Integration Center, Tohoku University from 2013 to 2016. He has been an assistant professor at the Department of Mechanical System Engineering, Graduate School of Engineering at Tohoku University since 2016. (inomata@nme.mech.tohoku.ac.jp)

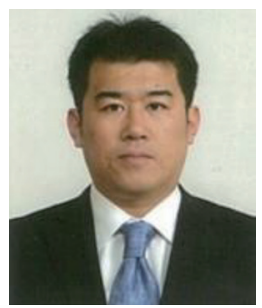

Zhuqing Wang received his Dr.Eng. degree from Yamanashi University, Kofu, Japan in 2011. He was an assistant professor at the Graduate School of Engineering, Tohoku University. His research interests cover wide areas, including micro-electromechanical systems (MEMS), nanoelectromechanical systems, and ultrasensitive sensing based on thermal biodevices for practical applications. (wzhuqing@nme.mech.tohoku.ac.jp)

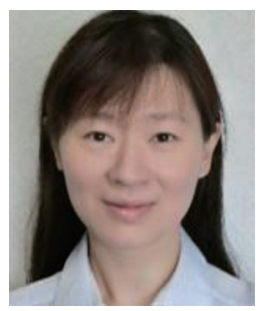

Yu-Ching Lin received her M.S. degree from National Taiwan University and then her Ph.D. degree from Tohoku University in 2006. She has worked as an assistant professor and then an associate professor at Tohoku University, Japan; a scientific staff member of Fraunhofer ENAS, Germany; a visiting scholar at UC Berkeley, USA; a technical adviser of MEMSCORE Corporation, Japan. Currently, she is Director of Advanced Microsystem Research Institute at Goertek Technology Japan.

(yclin@nme.mech.tohoku.ac.jp) 


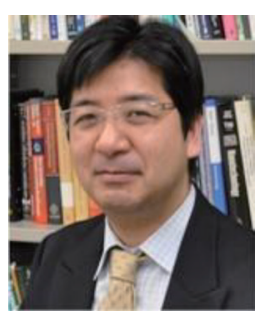

Takahito Ono received his D.E. degree in mechatronics and precision engineering from Tohoku University in 1996. From 1996 to 1999, he was a research associate in the Department of Mechatronics and Precision Engineering, Tohoku University. From 1999 to 2009, he was an associate professor. He is currently a professor at the Department of Mechanical Systems Engineering of Tohoku University and the Director of Microsystem Integration Center, Tohoku University. He was a professor at the Department of Mechanical Engineering, Graduate School of Engineering at The University of Tokyo from 2013 to 2016. He is Director of the Micro/Nano Machining Education Center, Tohoku University from 2018. His expertise is in the areas of microelectromechanical systems (MEMS), nanoelectromechanical systems (NEMSs), silicon-based nanofabrication, ultrasensitive sensing based on resonating devices, scanning probe technologies, and nanoprobe sensing for nanoscale science and engineering. His recent interests cover nanomaterials and their integration into microsystems for applications in IoT sensors, environment monitoring, biomedical sensors, nanoenergy, and scientific instrumentation. (ono@nme.mech.tohoku.ac.jp) 